\title{
Power of a meromorphic function that share a set with its derivative
}

\author{
Bikash Chakraborty
}

\begin{abstract}
In this article, we deal with the problem of the uniqueness of the power of a meromorphic function with its derivative counterpart sharing a set and thus improve our recent result under some constraints.
\end{abstract}

Mathematics Subject Classification (2010): 30D35.

Keywords: Meromorphic function, weighted set sharing, uniqueness.

\section{Introduction and Definitions}

In this article, we assume that readers are familiar with basic Nevanlinna theory $([6])$. By $\mathbb{C}$ and $\mathbb{N}$, we mean the set of complex numbers and the set of natural numbers respectively.

Let $f$ and $g$ be two non-constant meromorphic functions and let $a$ be a finite complex number. If $f-a$ and $g-a$ have the same zeros with the same multiplicities, then we say that $f$ and $g$ share the value $a$ in counting multiplicities (in short, CM). Similarly, we say that $f$ and $g$ share the value $a$ in ignoring multiplicities (in short, IM), provided that $f-a$ and $g-a$ have the same set of zeros, where the multiplicities are not taken into account.

Also, we say that $f$ and $g$ share $\infty$ CM (resp. IM), if $1 / f$ and $1 / g$ share $0 \mathrm{CM}$ (resp. IM).

Next we shortly recall the notion of weighted sharing which appeared in the literature in 2001 ([7]) as scaling between IM sharing to CM sharing.

Definition 1.1. ([7]) Let $k$ be a non-negative integer or infinity. For $a \in \mathbb{C} \cup\{\infty\}$, we denote by $E_{k}(a ; f)$, the set of all $a$-points of $f$, where an $a$-point of multiplicity $m$ is counted $m$ times if $m \leq k$ and $k+1$ times if $m>k$.

If $E_{k}(a ; f)=E_{k}(a ; g)$, we say that $f$ and $g$ share the value $a$ with weight $k$. 
We write $f, g$ share $(a, k)$ to mean that $f, g$ share the value $a$ with weight $k$. Clearly $f$ and $g$ share a value $a$ IM (resp. CM) if and only if $f$ and $g$ share $(a, 0)$ (resp. $(a, \infty))$.

Definition 1.2. ([7]) Let $S \subset \mathbb{C} \cup\{\infty\}$ and $k$ be a non-negative integer or $\infty$. We denote by $E_{f}(S, k)$, the set $\cup_{a \in S} E_{k}(a ; f)$.

If $E_{f}(S, k)=E_{g}(S, k)$, then we say $f, g$ share the set $S$ with weight $k$.

Definition 1.3. A set $S \subset \mathbb{C} \cup\{\infty\}$ is called a unique range set for meromorphic functions with weight $k$ (in short, $U R S M_{k}$ ), if for any two non-constant meromorphic functions $f$ and $g, E_{f}(S, k)=E_{g}(S, k)$ implies $f \equiv g$.

Similarly, one can define unique range set for entire functions with weight $k$ (in brief, $\left.U R S E_{k}\right)$.

Next we recall following two definitions:

Definition 1.4. ([2]) Let $z_{0}$ be a zero of $f-a$ of multiplicity $p$ and a zero of $g-a$ of multiplicity $q$.

i) We denote by $\bar{N}_{L}(r, a ; f)$, the counting function of those $a$-points of $f$ and $g$ where $p>q \geq 1$

ii) by $N_{E}^{1)}(r, a ; f)$, the counting function of those $a$-points of $f$ and $g$ where $p=q=1$ and

iii) by $\bar{N}_{E}^{(2}(r, a ; f)$, the counting function of those $a$-points of $f$ and $g$ where $p=q \geq$ 2 , each point in these counting functions is counted only once.

In the same way, we can define $\bar{N}_{L}(r, a ; g), N_{E}^{1)}(r, a ; g), \bar{N}_{E}^{(2}(r, a ; g)$.

Definition 1.5. ([2]) Let $f$ and $g$ share a value $a$ IM. We denote by $\bar{N}_{*}(r, a ; f, g)$, the reduced counting function of those $a$-points of $f$ whose multiplicities differ from the multiplicities of the corresponding $a$-points of $g$.

Clearly

$$
\bar{N}_{*}(r, a ; f, g) \equiv \bar{N}_{*}(r, a ; g, f) \quad \text { and } \quad \bar{N}_{*}(r, a ; f, g)=\bar{N}_{L}(r, a ; f)+\bar{N}_{L}(r, a ; g) \text {. }
$$

The subject of sharing values between entire functions and their derivatives was first studied by Rubel and Yang ([11]). In 1977, they proved that if non-constant entire functions $f$ and $f^{(1)}$ share two distinct finite numbers $a, b \mathrm{CM}$, then $f \equiv f^{(1)}$.

Later, in 1979, analogous result for IM sharing was obtained by Mues and Steinmetz $([10])$ in the following manner:

Theorem A. ([10]) Let $f$ be a non-constant entire function. If $f$ and $f^{(1)}$ share two distinct values $a, b$ IM, then $f \equiv f^{(1)}$.

In the direction of value sharing and uniqueness problem, Yang and Zhang ([12]) were the first authors to consider the uniqueness of a power of a meromorphic (resp. entire) function $F=f^{m}$ and its derivative $F^{(1)}$ as:

Theorem B. ([12]) Let $f$ be a non-constant entire (resp. meromorphic) function and $m>7$ (resp. 12) be an integer. If $F$ and $F^{(1)}$ share $1 \mathrm{CM}$, then $F=F^{(1)}$, and $f$ assumes the form

$$
f(z)=c e^{\frac{z}{m}},
$$


where $c$ is a nonzero constant.

In this direction, Zhang ([13]) further improved the above result in the following manner:

Theorem C. ([13]) Let $f$ be a non-constant entire function, $m, k$ be positive integers and $a(z)(\not \equiv 0, \infty)$ be a small function of $f$. Suppose $f^{m}-a$ and $\left(f^{m}\right)^{(k)}-a$ share the value $0 \mathrm{CM}$ and $m>k+4$. Then $f^{m} \equiv\left(f^{m}\right)^{(k)}$ and $f$ assumes the form

$$
f(z)=c e^{\frac{\lambda}{m} z}
$$

where $c$ is a nonzero constant and $\lambda^{k}=1$.

Afterwards, there were many improvements and generalizations concerning the uniqueness of $f^{m}$ and $\left(f^{m}\right)^{(k)}$. But all authors paid their attention on value sharing or small function sharing, not on set sharing problem. Thus the natural curiosity will be:

Question 1.1. Is it possible to change the value sharing notion into set sharing notion in Theorem $C$ keeping the conclusions same?

In connection to Question 1.1, recently we considered the uniqueness of $f$ and $f^{(k)}$ when they share a set $S$ instead of a value $a(\neq 0, \infty)$. To discuss the results in ([3]), we first introduce the polynomial of Lin and Yi ([8]).

$$
P(z)=a z^{n}-n(n-1) z^{2}+2 n(n-2) b z-(n-1)(n-2) b^{2},
$$

where $n \geq 3$ is an integer and $a$ and $b$ are two nonzero complex numbers satisfying $a b^{n-2} \neq 2$. Clearly the polynomial $P(z)$ has only simple zeros.

In $([3])$, we considered the uniqueness of $f$ and $f^{(k)}$ when they share a set.

Theorem E. ([3]) Let $n(\geq 8), k(\geq 1)$ be two positive integers and $f$ be a non-constant meromorphic function. Suppose that $S=\{z: P(z)=0\}$ where $P(z)$ is defined by (1.1). If $E_{f}(S, 3)=E_{f^{(k)}}(S, 3)$, then $f \equiv f^{(k)}$.

But in this paper, we will see that if we impose some restrictions on $f$, then the cardinality of the set $S$ defined in Theorem $\mathrm{E}$ will be reduced remarkably.

Thus our main goal is to reduce the cardinality of this particular set $S$ and to establish the uniqueness of the power of a meromorphic function with its derivative counterpart sharing the set $S$.

The method of proving of the main result of this paper is from $([3,4])$.

\section{Main Result}

Theorem 2.1. Let $f$ be a non-constant meromorphic function and $n(\geq 6), k(\geq 1)$ and $m(\geq k+1)$ be three positive integers. Suppose that $S=\{z: P(z)=0\}$ where $P(z)$ is defined by (1.1). If $E_{f^{m}}(S, 3)=E_{\left(f^{m}\right)^{(k)}}(S, 3)$, then $f^{m} \equiv\left(f^{m}\right)^{(k)}$ and hence $f$ takes the form

where $c$ is a non-zero constant and $\zeta^{k}=1$.

$$
f(z)=c e^{\frac{\zeta}{m} z}
$$


The following example shows that for a non-constant entire function the set $S$ in Theorem 2.1 can not be replaced by an arbitrary set containing six distinct elements.

Example 2.1. ([3]) For a non-zero complex number $a$, let

$$
S=\left\{a \omega, a \sqrt{\omega}, a, \frac{a}{\sqrt{\omega}}, \frac{a}{\omega}, \frac{a}{\omega \sqrt{\omega}}\right\},
$$

where $\omega$ is the non-real cubic root of unity. Choosing

$$
f(z)=e^{\frac{\omega^{\frac{1}{2 k}}}{m} z}
$$

(taking the principal branch when $m \geq 2$ ), it is easy to verify that $f^{m}$ and $\left(f^{m}\right)^{(k)}$ share $(S, \infty)$, but $f^{m} \not \equiv\left(f^{m}\right)^{(k)}$

Remark 2.1. However the following questions are still unknown to us:

i) Is it possible to omit the condition $m \geq k+1$ keeping the condition $n(\geq 6)$ same in Theorem 2.1?

ii) Under the same conditions of Theorem 2.1, is it possible to further reduce the cardinality of $S$ ?

\section{Auxiliary Lemmas}

Before going to discuss the necessary lemmas, we recall a well known auxiliary function as

$$
H=\left(\frac{F^{\prime \prime}}{F^{\prime}}-\frac{2 F^{\prime}}{F-1}\right)-\left(\frac{G^{\prime \prime}}{G^{\prime}}-\frac{2 G^{\prime}}{G-1}\right),
$$

where $F:=R\left(f^{m}\right), G:=R\left(\left(f^{m}\right)^{(k)}\right)$ and the expression

$$
\frac{a(*)^{n}}{n(n-1)\left(*-\alpha_{1}\right)\left(*-\alpha_{2}\right)}
$$

is denoted by $R(*)$.

In addition, in the expression of $R(*)$, we choose $\alpha_{1}$ and $\alpha_{2}$ as the distinct roots of the equation

$$
n(n-1) z^{2}-2 n(n-2) b z+(n-1)(n-2) b^{2}=0 .
$$

Lemma 3.1. ([1]) Let $Q(z)=(n-1)^{2}\left(z^{n}-1\right)\left(z^{n-2}-1\right)-n(n-2)\left(z^{n-1}-1\right)^{2}$. Then

$$
Q(z)=(z-1)^{4} \prod_{i=1}^{2 n-6}\left(z-\beta_{i}\right)
$$

where $\beta_{i} \in \mathbb{C} \backslash\{0,1\}(i=1,2, \ldots, 2 n-6)$, which are distinct.

Lemma 3.2. Let $F$ and $G$ share $(1, l)$ where $F$ and $G$ defined as earlier, then

i) $\bar{N}_{L}(r, 1 ; F) \leq \mu(\bar{N}(r, 0 ; f)+\bar{N}(r, \infty ; f))+S(r, f)$,

ii) $\bar{N}_{L}(r, 1 ; G) \leq \mu\left(\bar{N}\left(r, 0 ;\left(f^{m}\right)^{k}\right)+\bar{N}(r, \infty ; f)\right)+S(r, f)$, where $\mu=\min \left\{\frac{1}{l}, 1\right\}$. 
Proof. The proofs are similar to the proof of Lemma 2.2 of ([3]). So we omit the details.

Lemma 3.3. Suppose that $F$ and $G$ share $(1, l)$ and $F \not \equiv G$. If $m \geq k+1$, then

$$
\bar{N}(r, 0 ; f) \leq \bar{N}\left(r, 0,\left(f^{m}\right)^{(k)}\right) \leq \frac{2 \mu+1}{\eta-2 \mu} \bar{N}(r, \infty ; f)+\frac{2}{\eta-2 \mu} T(r)+S(r),
$$

where $T(r)=T\left(r, f^{m}\right)+T\left(r,\left(f^{m}\right)^{(k)}\right), S(r)=S(r, f)$ and $\eta=(m-k) n-1$.

Proof. For the proof, we define $U:=\frac{F^{\prime}}{(F-1)}-\frac{G^{\prime}}{(G-1)}$ and consider two cases:

Case 1. Assume $U \equiv 0$. Then by integration, we get

$$
F-1=B(G-1) \text {. }
$$

If $z_{0}$ is a zero of $f$, then $B=1$ which is impossible, thus $\bar{N}(r, 0 ; f)=S(r, f)$. Hence the result holds.

Case 2. Next we assume that $U \not \equiv 0$.

If $z_{0}$ is a zero of $f$ of order $t$, then it is a zero of $F$ of order $m t n$ and that of $G$ is of order $(m t-k) n$. Hence $z_{0}$ is a zero of $U$ of order at least $\eta=(m-k) n-1$. Thus

$$
\begin{aligned}
\bar{N}(r, 0 ; f) \leq & \bar{N}\left(r, 0,\left(f^{m}\right)^{(k)}\right) \\
\leq & \frac{1}{\eta} N(r, 0 ; U) \leq \frac{1}{\eta} N(r, \infty ; U)+S(r, f) \\
\leq & \frac{1}{\eta}\left\{\bar{N}_{L}(r, 1 ; F)+\bar{N}_{L}(r, 1 ; G)+\bar{N}_{L}(r, \infty ; F)+\bar{N}_{L}(r, \infty ; G)\right. \\
& +\bar{N}(r, \infty ; G \mid F \neq \infty)+\bar{N}(r, \infty ; F \mid G \neq \infty)\}+S(r, f) \\
\leq & \frac{1}{\eta}\left\{\mu\left(\bar{N}(r, 0 ; f)+\bar{N}\left(r, 0 ;\left(f^{m}\right)^{(k)}\right)+2 \bar{N}(r, \infty ; f)\right)\right. \\
& +\bar{N}(r, \infty ; f)+\bar{N}\left(r, \alpha_{1} ; f^{m}\right)+\bar{N}\left(r, \alpha_{2} ; f^{m}\right) \\
& +\bar{N}\left(r, \alpha_{1} ;\left(f^{m}\right)^{(k)}\right)+\bar{N}\left(r, \alpha_{2} ;\left(f^{m}\right)^{(k)}\right\}+S(r, f) \\
\leq & \frac{1}{\eta}\left\{2 \mu \bar{N}\left(r, 0 ;\left(f^{m}\right)^{(k)}\right)+(2 \mu+1) \bar{N}(r, \infty ; f)+2 T(r)\right\}+S(r) .
\end{aligned}
$$

Thus

$$
\bar{N}(r, 0 ; f) \leq \bar{N}\left(r, 0,\left(f^{m}\right)^{(k)}\right) \leq \frac{2 \mu+1}{\eta-2 \mu} \bar{N}(r, \infty ; f)+\frac{2}{\eta-2 \mu} T(r)+S(r) .
$$

Hence the proof of the lemma is completed.

Lemma 3.4. Let $F$ and $G$ share $(1, l)$ and $F \not \equiv G$. If $m \geq k+1$, then

$$
\bar{N}(r, \infty ; f) \leq \frac{\mu(\eta-2 \mu)+2}{(\lambda-2 \mu)(\eta-2 \mu)-(2 \mu+1)} T(r)+S(r),
$$

where $T(r)=T\left(r, f^{m}\right)+T\left(r,\left(f^{m}\right)^{(k)}\right)$ and $S(r)=S(r, f), \lambda=m(n-2)-1$ and $\mu=\min \left\{\frac{1}{l}, 1\right\}$. 
Proof. For the proof, we define $V:=\frac{F^{\prime}}{F(F-1)}-\frac{G^{\prime}}{G(G-1)}$ and consider two cases:

Case 1. Assume that $V \equiv 0$. Then by integration, we get

$$
\left(1-\frac{1}{F}\right)=A\left(1-\frac{1}{G}\right) \text {. }
$$

As $f^{m}$ and $\left(f^{m}\right)^{(k)}$ share $(\infty, 0)$, so if $\bar{N}(r, \infty ; f) \neq S(r, f)$, then $A=1$, i.e., $F=G$, which is not possible. So $\bar{N}(r, \infty ; f)=S(r, f)$. Thus the lemma holds.

Case 2. Next we assume that $V \not \equiv 0$.

If $z_{0}$ is a pole of $f$ of order $p$, then it is a pole of $\left(f^{m}\right)^{(k)}$ of order $(p m+k)$ and that of $F$ and $G$ are $p m(n-2)$ and $(p m+k)(n-2)$ respectively.

Hence $z_{0}$ is a zero of $\left(\frac{F^{\prime}}{F-1}-\frac{F^{\prime}}{F}\right)$ of order at least $p m(n-2)-1$ and a zero of $V$ of order at least $\lambda$ where $\lambda=m(n-2)-1$.

Since the zeros of $F$ comes from zeros of $f^{m}$ and that of $G$ comes from zeros of $\left(f^{m}\right)^{(k)}$, so for the points where $f=0$, each zero of $F$ will be of larger multiplicities than that of $G$. Consequently

$$
\begin{aligned}
& \bar{N}_{*}(r, 0 ; F, G)+\bar{N}(r, 0 ; G \mid F \neq 0) \\
\leq & \bar{N}_{L}(r, 0 ; F)+\bar{N}_{L}(r, 0 ; G)+\bar{N}(r, 0 ; G \mid F \neq 0) \\
\leq & \bar{N}_{L}(r, 0 ; F)+\bar{N}(r, 0 ; G \mid F \neq 0) \leq \bar{N}(r, 0 ; G) .
\end{aligned}
$$

Thus

$$
\begin{aligned}
\bar{N}(r, \infty ; f) \leq & \frac{1}{\lambda} N(r, 0 ; V) \\
\leq & \frac{1}{\lambda} N(r, \infty ; V)+S(r, f) \\
\leq & \frac{1}{\lambda}\left\{\bar{N}_{L}(r, 1 ; F)+\bar{N}_{L}(r, 1 ; G)+\bar{N}_{L}(r, 0 ; F)\right. \\
& +\bar{N}_{L}(r, 0 ; G)+\bar{N}(r, 0 ; G \mid F \neq 0\}+S(r, f) \\
\leq & \frac{1}{\lambda}\left\{\bar{N}_{L}(r, 1 ; F)+\bar{N}_{L}(r, 1 ; G)+\bar{N}(r, 0 ; G)\right\}+S(r, f) \\
\leq & \frac{1}{\lambda}\left\{\mu\left(\bar{N}(r, 0 ; f)+\bar{N}\left(r, 0 ;\left(f^{m}\right)^{(k)}\right)+2 \bar{N}(r, \infty ; f)\right)\right. \\
& \left.+\bar{N}\left(r, 0 ;\left(f^{m}\right)^{(k)}\right)\right\}+S(r, f) .
\end{aligned}
$$

Now using Lemma 3.3, we get

$$
\begin{aligned}
(\lambda-2 \mu) \bar{N}(r, \infty ; f) & \leq \mu T(r)+\bar{N}\left(r, 0 ;\left(f^{m}\right)^{(k)}\right)+S(r) \\
& \leq \mu T(r)+\frac{2 \mu+1}{\eta-2 \mu} \bar{N}(r, \infty ; f)+\frac{2}{\eta-2 \mu} T(r)+S(r) .
\end{aligned}
$$

Thus

$$
\bar{N}(r, \infty ; f) \leq \frac{\mu(\eta-2 \mu)+2}{(\lambda-2 \mu)(\eta-2 \mu)-(2 \mu+1)} T(r)+S(r) .
$$

Hence the proof is completed. 
Lemma 3.5. If $H \not \equiv 0$ and $F$ and $G$ share $(1, l)$ then

$$
\begin{aligned}
& N(r, \infty ; H) \\
\leq & \bar{N}(r, \infty ; f)+\bar{N}\left(r, 0 ;\left(f^{m}\right)^{(k)}\right)+\bar{N}\left(r, b ; f^{m}\right)+\bar{N}\left(r, b ;\left(f^{m}\right)^{(k)}\right) \\
& +\bar{N}_{L}(r, 1 ; F)+\bar{N}_{L}(r, 1 ; G)+\bar{N}_{0}\left(r, 0 ;\left(f^{m}\right)^{\prime}\right)+\bar{N}_{0}\left(r, 0 ;\left(f^{m}\right)^{(k+1)}\right),
\end{aligned}
$$

where $\bar{N}_{0}\left(r, 0 ;\left(f^{m}\right)^{\prime}\right)$ denotes the counting function of the zeros of $\left(f^{m}\right)^{\prime}$ which are not the zeros of $f\left(f^{m}-b\right)$ and $F-1$. Similarly $\bar{N}_{0}\left(r, 0 ;\left(f^{m}\right)^{(k+1)}\right)$ is defined.

Proof. The proof is obvious if we are keeping the followings in our mind:

As zeros of $F$ come from the zeros of $f^{m}$ and that of $G$ come from the zeros of $\left(f^{m}\right)^{(k)}$, so $\bar{N}(r, 0 ; F) \leq \bar{N}(r, 0 ; G)$ when $m \geq k+1$. Also

$$
\bar{N}(r, \infty ; F) \leq \bar{N}\left(r, \infty ; f^{m}\right)+\bar{N}\left(r, \alpha_{1} ; f^{m}\right)+\bar{N}\left(r, \alpha_{2} ; f^{m}\right) .
$$

Again simple zeros of $f^{m}-\alpha_{i}$ are not poles of $H$ and multiple zeros of $f^{m}-\alpha_{i}$ are zeros of $\left(f^{m}\right)^{\prime}$.

Similar explanation for $G$ also holds.

Lemma 3.6. If $F \equiv G$ and $n>5$, then $f^{m}=\left(f^{m}\right)^{(k)}$, i.e., $f$ takes the form

$$
f(z)=c e^{\frac{\zeta}{m} z}
$$

where $c$ is a non zero constant and $\zeta^{k}=1$.

Proof. Given $F \equiv G$, that is,

$$
\begin{aligned}
& n(n-1) f^{2 m}\left(\left(f^{m}\right)^{(k)}\right)^{2}\left\{f^{(n-2) m}-\left(\left(f^{m}\right)^{(k)}\right)^{n-2}\right\} \\
& -2 n(n-2) b f^{m}\left(f^{m}\right)^{(k)}\left\{f^{(n-1) m}-\left(\left(f^{m}\right)^{(k)}\right)^{n-1}\right\} \\
& +(n-1)(n-2) b^{2}\left\{\left(f^{m}\right)^{n}-\left(\left(f^{m}\right)^{(k)}\right)^{n}\right\}=0 .
\end{aligned}
$$

By substituting $h=\frac{\left(f^{m}\right)^{(k)}}{f^{m}}$ in above, we get

$$
\begin{aligned}
n(n-1) h^{2} f^{2 m}\left(h^{n-2}-1\right) & -2 n(n-2) b h f^{m}\left(h^{n-1}-1\right) \\
& +(n-1)(n-2) b^{2}\left(h^{n}-1\right)=0 .
\end{aligned}
$$

If $h$ is a non-constant meromorphic function, then by Lemma 3.1, we get

$$
\begin{aligned}
& \left\{n(n-1) h f^{m}\left(h^{n-2}-1\right)-n(n-2) b\left(h^{n-1}-1\right)\right\}^{2} \\
& =-n(n-2) b^{2}(h-1)^{4} \prod_{i=1}^{2 n-6}\left(h-\beta_{i}\right) .
\end{aligned}
$$

Then by the second fundamental theorem, we get

$$
\begin{aligned}
& (2 n-6) T(r, h) \\
\leq & \bar{N}(r, \infty ; h)+\bar{N}(r, 0 ; h)+\sum_{i=1}^{2 n-6} \bar{N}\left(r, 0 ; h-\beta_{i}\right)+S(r, h) \\
\leq & \bar{N}\left(r, \infty ; h+\bar{N}(r, 0 ; h)+\frac{1}{2} \sum_{i=1}^{2 n-6} N\left(r, 0 ; h-\beta_{i}\right)+S(r, h)\right. \\
\leq & (n-1) T(r, h)+S(r, h),
\end{aligned}
$$


which is a contradiction as $n>5$.

Thus $h$ is a constant. Hence as $f$ is non-constant and $b \neq 0$, we get from (3.4) that

$$
\left(h^{n-2}-1\right)=0,\left(h^{n-1}-1\right)=0 \text { and }\left(h^{n}-1\right)=0 .
$$

That is, $h=1$. Consequently $f^{m}=\left(f^{m}\right)^{(k)}$.

If $f^{m}=\left(f^{m}\right)^{(k)}$, then we claim that 0 and $\infty$ are the Picard exceptional value of $f$.

For the proof, if $z_{0}$ is a zero of $f$ of order $t$, then it is a zero of $f^{m}$ and $\left(f^{m}\right)^{(k)}$ of order $m t$ and $(m t-k)$ respectively, which is impossible.

Again if $z_{0}$ is a pole of $f$ of order $s$, then it is a pole of $f^{m}$ and $\left(f^{m}\right)^{(k)}$ of order $m s$ and $(m s+k)$ respectively, which is impossible.

Thus our claim is true and hence $f$ takes the form of

$$
f(z)=c e^{\frac{\zeta}{m} z},
$$

where $c$ is a non zero constant and $\zeta^{k}=1$.

Lemma 3.7. If $H \equiv 0$ and $n>5$, then $f^{m}=\left(f^{m}\right)^{(k)}$.

Proof. Since $H \equiv 0$, on integration, we have

$$
F=\frac{A G+B}{C G+D}
$$

where $A, B, C, D$ are constant satisfying $A D-B C \neq 0$, and $F$ and $G$ share $(1, \infty)$.

Thus applying Mokhon'ko's Lemma $([9])$ in (3.5), we get

$$
T\left(r, f^{m}\right)=T\left(r,\left(f^{m}\right)^{(k)}\right)+S(r, f) .
$$

Again from (3.5), we get $\bar{N}(r, \infty ; f)=S(r, f)$ if $C \neq 0$, otherwise $f^{m}$ and $\left(f^{m}\right)^{(k)}$ share $(\infty, \infty)$ if $C=0$.

As $A D-B C \neq 0$, so $A=C=0$ never occurs. Thus we consider the following cases:

Case 1. If $A C \neq 0$, then

$$
F-\frac{A}{C}=\frac{B C-A D}{C(C G+D)}
$$

So,

$$
\bar{N}\left(r, \frac{A}{C} ; F\right)=\bar{N}(r, \infty ; G) .
$$

Now by using the second fundamental theorem, we get

$$
\begin{aligned}
& T(r, F) \\
\leq & \bar{N}(r, \infty ; F)+\bar{N}(r, 0 ; F)+\bar{N}\left(r, \frac{A}{C} ; F\right)+S(r, F) \\
\leq & \bar{N}(r, \infty ; f)+\bar{N}\left(r, \alpha_{1} ; f^{m}\right)+\bar{N}\left(r, \alpha_{2} ; f^{m}\right)+\bar{N}\left(r, 0 ; f^{m}\right) \\
& +\bar{N}\left(r, \infty ;\left(f^{m}\right)^{(k)}\right)+\bar{N}\left(r, \alpha_{1} ;\left(f^{m}\right)^{(k)}\right)+\bar{N}\left(r, \alpha_{2} ;\left(f^{m}\right)^{(k)}\right)+S(r, f) \\
\leq & \frac{5}{n} T(r, F)+S(r, F),
\end{aligned}
$$


which is a contradiction as $n>5$.

Case 2. Next we consider $A C=0$. Then obviously $A=0$ and $C=0$ can't occur. Thus we consider the following two subcases:

Subcase 2.1. If $A=0$ and $C \neq 0$, then obviously $B \neq 0$ and

$$
F=\frac{1}{\gamma G+\delta}
$$

where $\gamma=\frac{C}{B}$ and $\delta=\frac{D}{B}$.

If $F$ has no 1-point, then by using the second fundamental theorem, we get

$$
\begin{aligned}
& T(r, F) \\
\leq & \bar{N}(r, \infty ; F)+\bar{N}(r, 0 ; F)+\bar{N}(r, 1 ; F)+S(r, F) \\
\leq & \bar{N}\left(r, \infty ; f^{m}\right)+\bar{N}\left(r, \alpha_{1} ; f^{m}\right)+\bar{N}\left(r, \alpha_{2} ; f^{m}\right)+\bar{N}\left(r, 0 ; f^{m}\right)+S(r, f) \\
\leq & \frac{3}{n} T(r, F)+S(r, F),
\end{aligned}
$$

which is a contradiction as $n>5$. Thus $\gamma+\delta=1$ and $\gamma \neq 0$. So,

$$
F=\frac{1}{\gamma G+1-\gamma} .
$$

Consequently, $\bar{N}\left(r, 0 ; G+\frac{1-\gamma}{\gamma}\right)=\bar{N}(r, \infty ; F)$.

Now if $\gamma \neq 1$, then applying the second fundamental theorem and equation (3.6), we get

$$
\begin{aligned}
& T(r, G) \\
\leq & \bar{N}(r, \infty ; G)+\bar{N}(r, 0 ; G)+\bar{N}\left(r, 0 ; G+\frac{1-\gamma}{\gamma}\right)+S(r, G) \\
\leq & \bar{N}\left(r, \infty ;\left(f^{m}\right)^{(k)}\right)+\bar{N}\left(r, \alpha_{1} ;\left(f^{m}\right)^{(k)}\right)+\bar{N}\left(r, \alpha_{2} ;\left(f^{m}\right)^{(k)}\right) \\
+ & \bar{N}\left(r, 0 ;\left(f^{m}\right)^{(k)}\right)+\bar{N}\left(r, \infty ;\left(f^{m}\right)\right)+\bar{N}\left(r, \alpha_{1} ;\left(f^{m}\right)\right) \\
+ & \bar{N}\left(r, \alpha_{2} ;\left(f^{m}\right)\right)+S(r, f) \\
\leq & \frac{5}{n} T(r, G)+S(r, G),
\end{aligned}
$$

which is a contradiction as $n>5$. Thus $\gamma=1$ and hence $F G \equiv 1$ which give

$$
f^{m n}\left(\left(f^{m}\right)^{(k)}\right)^{n}=\frac{n^{2}(n-1)^{2}}{a^{2}}\left(f^{m}-\alpha_{1}\right)\left(f^{m}-\alpha_{2}\right)\left(\left(f^{m}\right)^{(k)}-\alpha_{1}\right)\left(\left(f^{m}\right)^{(k)}-\alpha_{2}\right) .
$$

It is clear from the above equation that $f$ has no pole, because $n>5$. Now let $z_{0}$ be a $\alpha_{1 i}$ point of $f$ of order $s$, where $\left(\alpha_{1 i}\right)^{m}=\alpha_{1}$, then it can't be a pole of $\left(f^{m}\right)^{(k)}$ as $f$ has no pole, so $z_{0}$ is a zero of $\left(f^{m}\right)^{(k)}$ of order $q$ satisfying $n \leq n q=s$. Thus

$$
\begin{aligned}
\bar{N}(r, \infty ; f) & =S(r, f), \\
\bar{N}\left(f, \alpha_{1 i} ; f\right) & \leq \frac{1}{n} N\left(f, \alpha_{1 i} ; f\right) \text { and } \\
\bar{N}\left(f, \alpha_{2 j} ; f\right) & \leq \frac{1}{n} N\left(f, \alpha_{2 j} ; f\right) .
\end{aligned}
$$


Thus by the second fundamental theorem, we get

$$
\begin{aligned}
& (2 m-1) T(r, f) \\
\leq & \bar{N}(r, \infty ; f)+\sum_{i=1}^{m} \bar{N}\left(r, \alpha_{1 i} ; f\right)+\sum_{j=1}^{m} \bar{N}\left(r, \alpha_{2 j} ; f\right)+S(r, f) \\
\leq & \frac{2 m}{n} T(r, f)+S(r, f),
\end{aligned}
$$

which is not possible as $n>5$.

Subcase 2.2. If $A \neq 0$ and $C=0$, then obviously $D \neq 0$ and

$$
F=\lambda G+\mu,
$$

where $\lambda=\frac{A}{D}$ and $\mu=\frac{B}{D}$. If $F$ has no 1-point, then we arrive at a contradiction as the previous case. Thus $\lambda+\mu=1$ with $\lambda \neq 0$. Also

$$
\bar{N}\left(r, 0 ; G+\frac{1-\lambda}{\lambda}\right)=\bar{N}(r, 0 ; F) .
$$

Now if $\lambda \neq 1$, then by using the second fundamental theorem and equation (3.6), we get

$$
\begin{aligned}
& T(r, G) \\
\leq & \bar{N}(r, \infty ; G)+\bar{N}(r, 0 ; G)+\bar{N}\left(r, 0 ; G+\frac{1-\lambda}{\lambda}\right)+S(r, G) \\
\leq & \bar{N}\left(r, \infty ;\left(f^{m}\right)^{(k)}\right)+\bar{N}\left(r, \alpha_{1} ;\left(f^{m}\right)^{(k)}\right)+\bar{N}\left(r, \alpha_{2} ;\left(f^{m}\right)^{(k)}\right) \\
+ & \bar{N}\left(r, 0 ;\left(f^{m}\right)^{(k)}\right)+\bar{N}\left(r, 0 ;\left(f^{m}\right)\right)+S(r, f) \\
\leq & \frac{5}{n} T(r, G)+S(r, G),
\end{aligned}
$$

which is a contradiction as $n>5$. Thus $\lambda=1$ and hence $F \equiv G$.

Now in view of Lemma 3.6, we get $f^{m}=\left(f^{m}\right)^{(k)}$ as $n>5$, i.e. $f$ takes the form

$$
f(z)=c e^{\frac{\zeta}{m} z}
$$

where $c$ is a non zero constant and $\zeta^{k}=1$.

\section{Proof of Main Result}

Proof of Theorem 2.1 . Let $H$ be defined by equation (3.1). Now we consider two cases:

Case 1. First we assume $H \not \equiv 0$. Then clearly $F \not \equiv G$ and

$$
\bar{N}(r, 1 ; F \mid=1)=\bar{N}(r, 1 ; G \mid=1) \leq N(r, \infty ; H) .
$$


So by the second fundamental theorem and Lemma 3.5, we get

$$
\begin{aligned}
& (n+1) T\left(r, f^{m}\right) \\
\leq & \bar{N}(r, \infty ; f)+\bar{N}(r, 0 ; f)+\bar{N}\left(r, b ; f^{m}\right) \\
& +\bar{N}(r, 1 ; F)-N_{0}\left(r, 0,\left(f^{m}\right)^{\prime}\right)+S(r, f) \\
\leq \quad & 2\left\{\bar{N}(r, \infty ; f)+\bar{N}\left(r, b ; f^{m}\right)\right\}+\bar{N}\left(r, 0 ;\left(f^{m}\right)^{(k)}\right) \\
& +\bar{N}(r, 0 ; f)+\bar{N}\left(r, b ;\left(f^{m}\right)^{(k)}\right)+\bar{N}(r, 1 ; F \mid \geq 2) \\
& +\bar{N}_{L}(r, 1 ; F)+\bar{N}_{L}(r, 1 ; G)+\bar{N}_{0}\left(r, 0 ;\left(f^{m}\right)^{(k+1)}\right)+S(r, f) .
\end{aligned}
$$

Now

$$
\begin{aligned}
& \bar{N}(r, 1 ; F \mid \geq 2)+\bar{N}_{*}(r, 1 ; F, G)+\bar{N}_{0}\left(r, 0 ;\left(f^{m}\right)^{(k+1)}\right) \\
\leq & \bar{N}(r, 1 ; G \mid \geq 2)+\bar{N}(r, 1 ; G \mid \geq 3)+\bar{N}_{0}\left(r, 0 ;\left(f^{m}\right)^{(k+1)}\right) \\
\leq & N\left(r, 0 ;\left(f^{m}\right)^{(k+1)} \mid\left(f^{m}\right)^{(k)} \neq 0\right)+S(r, f) \\
\leq & \bar{N}\left(r, 0 ;\left(f^{m}\right)^{(k)}\right)+\bar{N}(r, \infty ; f)+S(r, f) .
\end{aligned}
$$

Thus

$$
\begin{aligned}
& (n+1) T\left(r, f^{m}\right) \\
\leq \quad & 2\left\{\bar{N}(r, \infty ; f)+\bar{N}\left(r, b ; f^{m}\right)\right\}+\bar{N}(r, 0 ; f) \\
& +2 \bar{N}\left(r, 0 ;\left(f^{m}\right)^{(k)}\right)+\bar{N}\left(r, b ;\left(f^{m}\right)^{(k)}\right)+\bar{N}(r, \infty ; f)+S(r, f) .
\end{aligned}
$$

Similarly for $\left(f^{m}\right)^{(k)}$, we get

$$
\begin{aligned}
& (n+1) T\left(r,\left(f^{m}\right)^{(k)}\right) \\
\leq \quad & 2\left\{\bar{N}(r, \infty ; f)+\bar{N}\left(r, b ;\left(f^{m}\right)^{(k)}\right)\right\}+\bar{N}\left(r, 0 ;\left(f^{m}\right)^{(k)}\right) \\
& +2 \bar{N}(r, 0 ; f)+\bar{N}\left(r, b ; f^{m}\right)+\bar{N}(r, \infty ; f)+S(r, f) .
\end{aligned}
$$

Adding (4.3) and (4.4), we get

$$
\begin{aligned}
(n+1) T(r) \leq & 6 \bar{N}(r, \infty ; f)+3\left\{\bar{N}(r, 0 ; f)+\bar{N}\left(r, 0 ;\left(f^{m}\right)^{(k)}\right)\right\} \\
& +3\left\{\bar{N}\left(r, b ; f^{m}\right)+\bar{N}\left(r, b ;\left(f^{m}\right)^{(k)}\right)\right\}+S(r, f)
\end{aligned}
$$

and

$$
(n-5) T(r) \leq 6 \bar{N}(r, \infty ; f)+S(r) .
$$

Thus using Lemma 3.4, we get

$$
(n-5) T(r) \leq \frac{6 \mu(\eta-2 \mu)+12}{(\lambda-2 \mu)(\eta-2 \mu)-(2 \mu+1)} T(r)+S(r),
$$

which is a contradiction as $n \geq 6$ and $l \geq 3$.

Case 2. Next we assume $H \equiv 0$. Then for $n>5$, applying Lemma 3.7, we have $f^{m}=\left(f^{m}\right)^{(k)}$. Thus by the same arguments using in Lemma 3.6, we see that $f$ takes the form

$$
f(z)=c e^{\frac{\zeta}{m} z}
$$


where $c$ is a non zero constant and $\zeta^{k}=1$. Thus the proof is completed.

Acknowledgement. The author is grateful to the referees for his/her valuable suggestions which considerably improved the presentation of the paper.

\section{References}

[1] Alzahary, T.C., Meromorphic functions with weighted sharing of one set, Kyungpook Math. J., 47(2007), no. 1, 57-68.

[2] Banerjee, A., Chakraborty, B., A new type of unique range set with deficient values, Afr. Mat., 26(2015), no. 7-8, 1561-1572.

[3] Banerjee, A., Chakraborty, B., Uniqueness of the power of meromorphic functions with its differential polynomial sharing a set, Math. Morav., 20(2016), no. 2, 1-14.

[4] Banerjee, A., Chakraborty, B., On the uniqueness of power of a meromorphic function sharing a set with its $k$-th derivative, J. Indian Math. Soc. (N.S.), 85(2018), no. 1-2, $1-15$.

[5] Chakraborty, B., Some aspects of uniqueness theory of entire and meromorphic functions, Ph.D. Thesis, arXiv: 1711.08808 [math.CV], 2017.

[6] Hayman, W.K., Meromorphic Functions, Clarendon Press, Oxford, 1964.

[7] Lahiri, I., Weighted sharing and uniqueness of meromorphic functions, Nagoya Math. J., 161(2001), 193-206.

[8] Lin, W.C., Yi, H.X., Uniqueness theorems for meromorphic functions that share three sets, Complex Var. Elliptic Equ., 48(2003), no. 4, 315-327.

[9] Mokhon'ko, A.Z., On the Nevanlinna characteristics of some meromorphic functions, Theory of Functions, Functional Analysis and Their Applications, Izd-vo Khar'kovsk, Un-ta, 14(1971), 83-87.

[10] Mues, E., Steinmetz, N., Meromorphe Funktionen, die mit ihrer Ableitung Werte teilen, Manuscripta Math., 29(1979), no. 2-4, 195-206.

[11] Rubel, L.A., Yang, C.C., Values shared by an entire function and its derivative, Complex Analysis (Proc. Conf., Univ. Kentucky, Lexington, Ky., 1976), 101-103; Lecture Notes in Math., 599, Springer, Berlin, 1977.

[12] Yang, L.Z., Zhang, J.L., Non-existence of meromorphic solutions of a Fermat type functional equation, Aequationes Math., 76(2008), no. 1-2, 140-150.

[13] Zhang, J.L., Meromorphic functions sharing a small function with their derivatives, Kyungpook Math. J., 49(2009), no. 1, 143-154.

[14] Zhang, J.L., Yang, L.Z., A power of a meromorphic function sharing a small function with its derivative, Ann. Acad. Sci. Fenn. Math., 34(2009), no. 1, 249-260.

Bikash Chakraborty

Ramakrishna Mission Vivekananda Centenary College

Department of Mathematics

Kolkata-700 118, India

e-mail: bikashchakraborty.math@yahoo.com, bikashchakrabortyy@gmail.com 